\title{
Autonomous Distributed Power Control for Cognitive Radio Networks
}

\author{
Sooyeol Im, Hyoungsuk Jeon, and Hyuckjae Lee \\ School of Engineering \\ Information and Communications University (ICU) \\ 119, Munjiro, Yuseong-gu, Daejeon, 305-732, Korea \\ Email: \{iamsyim, hschun, hjlee\}@icu.ac.kr
}

\begin{abstract}
In recent years, cognitive radio (CR) has received a great attention due to the ability to improve the spectrum utilization. It allows the secondary user (SU) in the CR network to opportunistically access the licensed spectrum of the primary user (PU). For this end, quality of service $(\mathrm{QoS})$ requirements for both the PU and the SU should be guaranteed at the same time. These QoSs can be respectively translated into the interference temperature at the primary receiver and the received signalto-interference-plus-noise-ratio (SINR) of the secondary receiver. In such a CR network, a power control can increase the energy efficiency by keeping the transmission power of the $\mathrm{SU}$ as low as possible within the QoS requirements.

In this paper, we propose an autonomous distributed power control scheme for CR networks that considers the QoS requirements of the $P U$ and the $S U$ simultaneously. Since the transmission power of each $\mathrm{SU}$ is constrained so that the interference temperature at the primary receiver caused by all SUs does not exceed the interference tolerance of the PU, the $\mathrm{QoS}$ requirement for the $\mathrm{PU}$ is always guaranteed. Through the simulation results, we demonstrate that the proposed scheme never exceed the interference tolerance of the PU.
\end{abstract}

Index Terms-Cognitive radio, quality of service, power control, distributed constrained power control, generalized distributed constrained power control

\section{INTRODUCTION}

In recent years, the demand for the spectrum resource is increasing rapidly with the emergence of various wireless services. Since there is only a finite amount of the spectrum resource, the remaining spectrum is being exhausted and it leads to the spectrum scarcity problem. The Federal Communications Commission (FCC) indicated that not all the spectrum is in use all of the time, and only about $30 \%$ of the spectrum is actually in use in certain given location and at certain time [1]. This led the FCC to revisit the current fixed spectrum management policy, and the FCC proposed that unlicensed devices flexibly utilize the TV spectrum with no harmful interference in 2004 [2], [3].

In this context, a cognitive radio (CR) is receiving a great attention due to the potential to realize the proposal of the FCC. In CR networks, the unlicensed device and the TV service correspond to the secondary user (SU) and the primary user (PU) respectively. It allows the $\mathrm{SU}$ in the $\mathrm{CR}$ network to opportunistically access the spectrum licensed to the PU without interfering with the PU. In other words, it senses the available spectrum and opportunistically utilizes the identified spectrum. Thus, CR has the ability to improve the spectrum utilization [4].

A power control which has been employed for improving the link performance in cellular networks can also be applied to $\mathrm{CR}$ networks. However the power control of CR networks is more complex, in that it should not only consider the quality of service (QoS) requirements of the SUs but also protect the PU communication link. Since the PU communication has priority over the SU communication, it is impossible for the SUs to share the PU channel deteriorating the link quality of the PU communication. Thus, SUs should always check the estimated interference at the primary receiver after determining their transmission power.

There are two categories in the power control for CR networks: centralized and distributed power control. For the centralized power control, a central manager controls the transmission power of all users within its coverage. A transmission power control system using Fuzzy Logic System (FLS) was proposed in [5]. With the built-in fuzzy power controller, the $\mathrm{SU}$ is able to dynamically adjust its transmission power in response to the changes of the interference level caused by the SU to the PU. In [6], considering the interference temperature constraints, the optimal power control problem was modeled as a concave minimization problem. In [5], [6], no SU is allowed to transmit before receiving authorization from the manager. Furthermore, all information required for managing the network should be known to the central entity, and very heavy signaling is inevitable.

For the distributed power control, on the other hand, each user controls its transmission power by itself using only local information. However, since the interference temperature at the primary receiver cannot be identified by the local information, it is difficult that the QoS requirement for the PU is guaranteed in the distributed power control. Thus, an additional process is needed to let the SUs recognize the interference temperature at the primary receiver [7], [8]. In [7], a joint coordination and power control (JCPC) algorithm was proposed. The optimal SU set is chosen in the coordination phase based on the game theory, and the transmission power of each SU in the set is distributively allocated in the power control phase. In [8], a genie-aided distributed power control algorithm was proposed. First, the transmission power of each SU is determined by 
the distributed power control algorithm. After that, if the interference level caused by all opportunistic communications is so high that the PU is violated, a genie placed near by the PU informs the SUs. Since an additional process is executed, they are not fully autonomous distributed power control algorithms.

In this paper, we propose an fully autonomous distributed power control scheme without an additional process for CR networks. Specifically, the constraint for the sum of the interference induced by all SUs in the network is replaced by new one which limits the individual transmission power. Since each SU determines its transmission power within the range of the limit to protect the PU, the additional process in previous works is unnecessary in our scheme. Thus, the proposed scheme easily realize the fully autonomous distributed power control algorithm. In this paper, the individual transmission power constraint and the proposed scheme are numerically derived, and the simulation results demonstrate that the proposed scheme never exceed the interference temperature limit (ITL) of the PU.

The rest of this paper is organized as follows. In section II, the system model used in this paper is introduced. Section III briefly reviews the distributed power control. In section IV, the proposed schemes are provided. In section V, the simulation environments are shown, and the results are analyzed. Finally, the conclusions are summarized in section VI.

\section{SySTEM MOdEL}

\section{A. Network Architecture}

As shown in Fig.1, we consider a CR network with one PU (TV) and $N$ SUs. In such a network topology, it is assumed that the spectrum is divided into non-overlapping orthogonal channels, and the TV and SUs are sharing one channel. Each user consists of a transmitting-receiving pair, and all SUs are located in a $h \times h$ square region. The distance between the center of the region and the primary receiver (TV receiver) is $d$. Each SU has a transmission range of $r$. The TV station communicates with a transmission power $P_{T V}$, and its effective transmission range is $R$. The TV receiver is located at the border of the TV coverage area which is the closest to the SUs.

We assume a path loss model that the transmission power only attenuates with distance. The path loss exponents of the $\mathrm{TV}$ and the SU are $\alpha_{1}$ and $\alpha_{2}$ respectively. Since the primary transmitter is a tall TV antenna while the SUs are located on the ground, the SU's transmission power attenuates faster with distance than the PU does. Thus, the path loss exponent of the $\mathrm{SU}$ is larger than that of the PU.

In such a CR network, the objective is to control the transmission powers of the secondary transmitters sharing the channel with the TV while guaranteeing the QoS requirements for both the PU and the SU simultaneously.

\section{B. Two QoS Requirements}

1) Interference Temperature for PU: Since the PU is the licensee of the channel, it should be able to communicate whenever it wants. Therefore, the total amount of interference

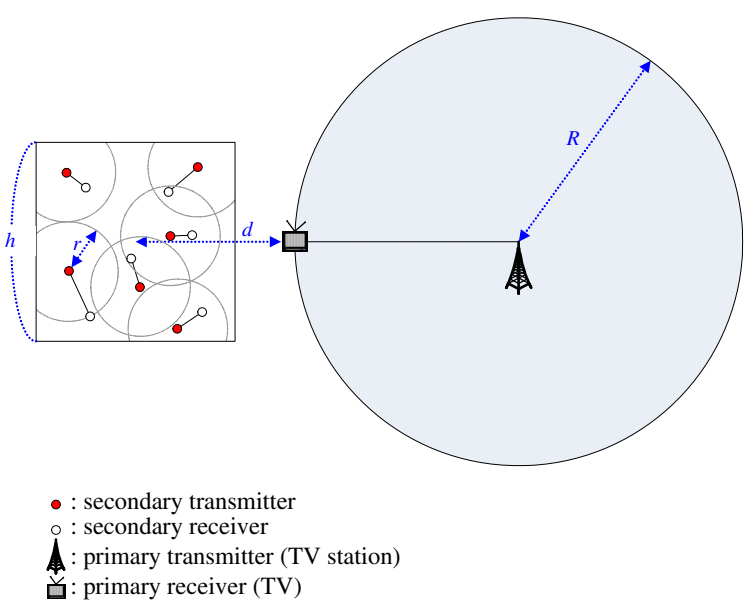

Fig. 1. Cognitive radio network architecture

at the primary receiver caused by all SUs' opportunistic communications should be less than the ITL of the PU. The ITL is the threshold value of the interference temperature which the PU can tolerate.

Let $G_{T V, i}$ be the link gain from the secondary transmitter $i$ to the TV receiver, and let $P_{i}$ be the transmission power of the secondary transmitter $i$. Then, the QoS requirement of the primary receiver is expressed as

$$
\xi_{T V}=\sum_{i=1}^{N} G_{T V, i} P_{i} \leq \xi_{T V}^{t h r}
$$

where $\xi_{T V}^{t h r}$ is the predefined ITL of the PU.

2) Received SINR of SU: For reliable communication, the received SINR of the SU should exceed the minimum required value.

Let $G_{i, j}$ be the link gain from the secondary transmitter $j$ to the secondary receiver $i$ and let $G_{i, T V}$ be the link gain from the TV transmitter to the secondary receiver $i$. Let $P_{T V}$ be the transmission power of the TV transmitter, and let $N_{0}$ be the receiver noise power. Then the QoS requirement of the secondary receiver $i$ is expressed as

$$
\gamma_{S U_{i}}=\frac{G_{i, i} P_{i}}{\sum_{j=1, j \neq i}^{N} G_{i, j} P_{j}+G_{i, T V} P_{T V}+N_{0}} \geq \gamma_{S U}^{t h r}
$$

where $\gamma_{S U}^{t h r}$ is the predefined threshold. Eq.(2) must be satisfied for all SUs.

\section{RELATED WORKS}

The inherent purpose of the power control is to keep the transmission power of the users in the network at the minimum level required to achieve the SINR for reliable communication. Thus, the efficiency of energy consumption can be maximized by minimizing the transmission power. 
From the Qos requirement for the SU, Eq.(2) can be rewritten as

$$
P_{i} \geq \gamma_{S U}^{t h r}\left(\sum_{j=1, j \neq i}^{N} \frac{G_{i, j}}{G_{i, i}} P_{j}+\frac{G_{i, T V}}{G_{i, i}} P_{T V}+\frac{N_{0}}{G_{i, i}}\right)
$$

which indicates the minimum transmission power of the SU $i$ required for reliable communication. To represent Eq.(3) using matrix notation, we define $N \times N$ matrix $\mathbf{H}$ such that

$$
h_{i, j}=\left\{\begin{array}{cc}
\gamma_{S U}^{t h r} \frac{G_{i, j}}{G_{i, i}}, & \text { for } i \neq j \\
0, & \text { for } i=j
\end{array},\right.
$$

and $N \times 1$ vector $\mathbf{U}$ such that

$$
u_{i}=\gamma_{S U}^{t h r} \frac{G_{i, T V} P_{T V}}{G_{i, i}}+\gamma_{S U}^{t h r} \frac{N_{0}}{G_{i, i}} .
$$

Then, the linear inequality given in Eq.(3) can be rewritten as $P_{i} \geq \sum_{j=1}^{N} h_{i, j} P_{j}+u_{i}$. Consequently, the matrix notation of the linear inequality is represented as

$$
(\mathbf{I}-\mathbf{H}) \mathbf{P} \geq \mathbf{U}
$$

where $\mathbf{I}$ is $N \times N$ identity matrix, and $\mathbf{P}$ is the transmission power vector $\mathbf{P}=\left(P_{1}, P_{2}, \ldots P_{N}\right)^{T}$.

If the maximum eigenvalue of matrix $\mathbf{H}$ is less than one, there exists a non-negative power vector $\mathbf{P}$ which satisfies Eq.(6). Therefore, the required SINR $\gamma_{S U}^{t h r}$ is achievable. In that case, the Pareto optimal power vector is

$$
\mathbf{P}^{*}=(\mathbf{I}-\mathbf{H})^{-1} \mathbf{U} \text {. }
$$

In practical implementation, however, the maximum transmission power is limited as

$$
0 \leq P_{i} \leq P_{S U}^{\max }, \text { for all } i \in\{1,2, \cdots, N\} .
$$

If the resulting transmission powers of Eq.(7) are within the range of Eq.(8), the transmission power allocation is feasible. To solve Eq.(7) distributively, a general iterative method was introduced in [9]. For the secondary transmitter $i$, the iterative power control method is

$$
\begin{aligned}
P_{i}(t+1) & =\frac{\gamma_{S U}^{t h r}}{G_{i, i}}\left(\sum_{j=1, j \neq i}^{N} G_{i, j} P_{j}(t)+G_{i, T V} P_{T V}+N_{0}\right) \\
& =\frac{\gamma_{S U}^{t h r}}{\gamma_{S U_{i}}(t)} P_{i}(t), \quad t=0,1, \cdots
\end{aligned}
$$

where $\gamma_{S U_{i}}(t)$ and $P_{i}(t)$ are the received SINR and the transmission power of the SU $i$ at the $t$ th iteration, respectively.

Because of the maximum transmission power limit, the iteration method is modified into power-limited form such as

$$
P_{i}(t+1)=\min \left\{\frac{\gamma_{S U}^{t h r}}{\gamma_{S U_{i}}(t)} P_{i}(t), P_{S U}^{\max }\right\}, \quad t=0,1, \cdots .
$$

This algorithm is DCPC [10]. The received SINR converges to $\gamma_{S U}^{t h r}$ distributively except for cases where the maximum transmission power $P_{S U}^{\max }$ is reached.
The drawback of DCPC is that the transmission power of the user reaches the maximum transmission power even if the user cannot achieve the minimum required SINR. Therefore, the user cannot communicate reliably even if it consumes the maximum transmission power. This causes the waste of energy and affects other users as an intense interference.

To complement the drawback of DCPC, GDCPC was introduced in [11]. When the user cannot achieve the required SINR, it reduces the power to arbitrary level within its transmission power range instead of necessarily using the maximum transmission power. This idea can save the energy consumption and decrease the interference to other users. Because the other users in the network take less interference, they can use higher transmission powers. As a result, the network can support more users than DCPC.

With above motivations, GDCPC can be represented as

$$
P_{i}(t+1)=\left\{\begin{array}{cl}
\frac{\gamma_{S U}^{t h r}}{\gamma_{S U_{i}}(t)} P_{i}(t), & \text { if } \frac{\gamma_{S U}^{t h r}}{\gamma_{S U_{i}}(t)} P_{i}(t) \leq P_{S U}^{\max } \\
\tilde{P}, & \text { if } \frac{\gamma_{S U}^{t h r}}{\gamma_{S U_{i}}(t)} P_{i}(t)>P_{S U}^{\max }
\end{array}\right.
$$

where the power value $\tilde{P}$ is chosen arbitrarily within the range of $0 \leq \tilde{P} \leq P_{S U}^{\max }$. The lower $\tilde{P}$ we choose, the less interference the user generates affecting the other users. If we set $\tilde{P}=P_{S U}^{\max }$, GDCPC becomes equal to DCPC.

\section{Autonomous Distributed Power Control for Cognitive Radio Networks}

Since DCPC and GDCPC do not consider the QoS requirement for the PU given by Eq.(1), the resulting transmission power of them may exceed the ITL of the primary receiver. To apply them to CR networks, therefore, an additional constraint should be considered. In [7], [8], an additional process for guaranteeing the constraint is executed in addition to the conventional power control algorithm. Thus, the power control cannot be executed at a time owing to the additional process.

In this section, two autonomous distributed power control schemes with no additional process for $\mathrm{CR}$ networks are proposed. Each SU executes DCPC or GDCPC considering the QoS requirement for the PU simultaneously. Since each SU controls its transmission power using only local information, it cannot know the other SUs' transmission power in the network. Thus, it is unattainable that each user knows the interference temperature at the primary receiver caused by all SUs including itself. In the proposed scheme, however, by translating the interference constraint by the sum of all SUs' transmission power to the individual constraint for each SU, the QoS requirement for the PU can be easily guaranteed.

The QoS requirement for the PU in Eq.(1) is guaranteed if the following condition

$$
G_{T V, i} P_{i} \leq \frac{\xi_{T V}^{t h r}}{N} \quad \text { for all } \quad i \in\{1,2, \cdots, N\}
$$

is satisfied. This means that the total interference constraint at the PU caused by all SUs is divided equally among SUs. 
From Eq.(12), therefore, the transmission power constraint of the SU $i$ to guarantee the PU can be represented as

$$
P_{i} \leq \frac{\xi_{T V}^{t h r}}{G_{T V, i} N}
$$

As seen in Eq.(13), however, each user should know the number of SUs in the network, the ITL of the PU, and the link gain from itself to the TV receiver. Furthermore, it is assumed that the network is stationary during the power control operation. Each SU can know the number of SUs by using ad-hoc routing protocol [12]. In the ad-hoc routing, the link information about all users belonging to the network is shared. To guarantee the priority of the PU, beaconing concepts were introduced in [13]. The primary receiver transmits the beacon including the information about the beacon power and the ITL of itself. Thus the SUs can know the link gain from itself to the TV receiver and the ITL of the TV receiver.

Since the transmission power of each SU is constrained so that the total interference caused by all SUs does not exceed the interference tolerance of PU, the QoS requirement for the $\mathrm{PU}$ is always guaranteed.

\section{A. Autonomous DCPC}

Since another maximum transmission power constraint is enforced to each user, the maximum transmission power of the $\mathrm{SU} i$ is written as

$$
P_{S U_{i}}^{\max }=\min \left\{P_{S U}^{\max }, \frac{\xi_{T V}^{t h r}}{G_{T V, i} N}\right\} .
$$

Consequently, the transmission power updating rule of the $\mathrm{SU}$ $i$ at the $t$ th iteration is written as

$P_{i}(t+1)=\min \left\{\frac{\gamma_{S U}^{t h r}}{\gamma_{S U_{i}}(t)} P_{i}(t), \min \left\{P_{S U}^{\max }, \frac{\xi_{T V}^{t h r}}{G_{T V, i} N}\right\}\right\}$.

\section{B. Autonomous GDCPC}

In the proposed GDCPC, like the proposed DCPC, another maximum transmission power constraint is enforced to each user to guarantee the QoS requirement for the PU. Thus, the maximum transmission power of the $\mathrm{SU} i$ is same as that of autonomous DCPC, and the updating rule of the $\mathrm{SU} i$ at the $t$ th iteration is written as

$$
P_{i}(t+1)=\left\{\begin{array}{cl}
\frac{\gamma_{S U}^{t h r}}{\gamma_{S U_{i}}(t)} P_{i}(t), & \text { if } \frac{\gamma_{S U}^{t h r}}{\gamma_{S U_{i}}(t)} P_{i}(t) \leq P_{S U_{i}}^{\max } \\
\tilde{P}_{i}, & \text { if } \frac{\gamma_{S U}^{t h r}}{\gamma_{S U_{i}}(t)} P_{i}(t)>P_{S U_{i}}^{\max }
\end{array}\right.
$$

where the arbitrary chosen power value is

$$
\begin{aligned}
\tilde{P}_{i} & =\min \left\{\tilde{P}, P_{S U_{i}}^{\max }\right\} \\
& =\min \left\{\tilde{P}, \min \left\{P_{S U}^{\max }, \frac{\xi_{T V}^{t h r}}{G_{T V, i} N}\right\}\right\} .
\end{aligned}
$$

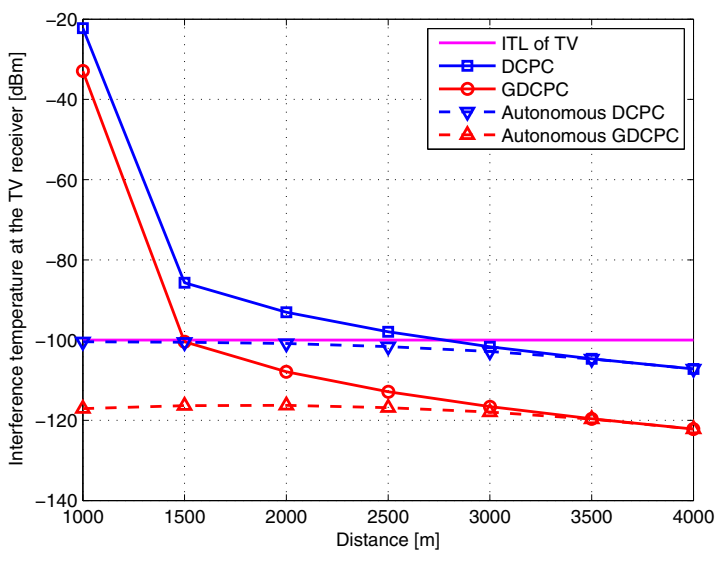

Fig. 2. Comparison of the interference temperature at the TV receiver

\section{Simulations}

In this section, some simulation results are provided to demonstrate the performance of the proposed schemes. We consider a CR network model shown in Fig.1.

There are $N=50$ transmitting-receiving pairs of SUs in a $2000 \mathrm{~m} \times 2000 \mathrm{~m}$ square region. The transmission range of the $\mathrm{SU}$ is $r=500 \mathrm{~m}$. The secondary transmitters are uniformly distributed in the square area, and the secondary receivers are located within the transmission range of the corresponding transmitter with a uniform distribution. The maximum transmission power of the $\mathrm{SU}$ is $P_{S U}^{\max }=100 \mathrm{~mW}$, and the transmission power of the TV station is $P_{T V}=100 \mathrm{~kW}$, and its effective transmission range is $R=70 \mathrm{~km}$. The receiver noise power is $N_{0}=10^{-11} \mathrm{~mW}$. The QoS requirements for both the PU and the SU are $\xi_{T V}^{t h r}=-100 \mathrm{dBm}$ and $\gamma_{S U}^{t h r}=3 \mathrm{~dB}$ respectively. The path loss exponents of the TV and the SU are 3 and 4 respectively. The initial transmission power of each SU is randomly chosen within the range of Eq.(8) with a uniform distribution. We chose the arbitrary power value $\tilde{P}=0 \mathrm{~mW}$ which is called GDCPC(I) [11].

In such a network, we varied $d$, which is the distance between the TV receiver and the center of the square region, from $1000 \mathrm{~m}$ to $4000 \mathrm{~m}$. It affects the interference temperature at the TV receiver. The longer $d$ is, the less the PU is interfered from the SU.

The interference temperature at the TV receiver caused by all opportunistic communications is shown in Fig.2. It demonstrates that the proposed schemes never exceed the ITL of the TV receiver. Since DCPC and GDCPC do not consider the PU, the interference temperatures exceed the ITL of the TV when the distance $d$ is short. Since GDCPC consumes less power than DCPC, the interference temperature for GDCPC is generally less than that for DCPC.

Fig.3 shows the number of SUs which can communicate reliably. In other words, the number of SUs whose received SINR is greater than or equal to the QoS requirement $\gamma_{S U}^{t h r}$ is shown. Since DCPC and GDCPC do not consider the PU, the number of SUs is is not influenced by the distance $d$. Thus, the 


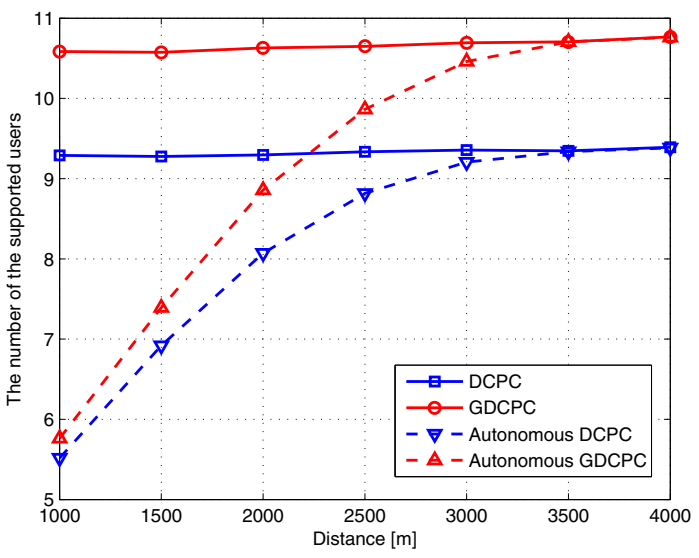

Fig. 3. Comparison of the number of the supported users

number of activated SUs for them is settled irrespective of the distance $d$. In the proposed schemes, there may be exist SUs whose transmission power constraint to protect the TV service is lower than the required level for reliable communication. Since the TV service should be protected first, the SUs cannot be activated in the network. Since GDCPC decreases the interference to other users, more SUs can communicate reliably than the case of DCPC.

If the distance $d$ is sufficiently long, the opportunistic communications scarcely affect to the TV receiver. As shown in Fig.2 and Fig.3, the results of the proposed schemes become equal to that of the conventional schemes, and the proposed schemes become unnecessary.

If the distance $d$ becomes sufficiently long, the link gain $G_{T V, i}$ becomes very small and the transmission power constraint $\frac{\xi_{T V}^{t h r}}{G_{T V, i} N}$ in Eq.(13) becomes very large. In Eq.(14), if the inequality

$$
P_{S U}^{\max } \leq \frac{\xi_{T V}^{t h r}}{G_{T V, i} N}
$$

is satisfied for all $i \in\{1,2, \cdots, N\}$, the proposed schemes become equal to the conventional schemes. Since the secondary transmitter nearest to the $\mathrm{TV}$ receiver is the worst interferer with the TV receiver, if the secondary transmitter satisfies Eq.(18), all secondary transmitters in the network satisfy that. Thus, if the distance $d$ satisfies the inequality

$$
d \geq\left(\frac{P_{S U}^{\max } N}{\xi_{T V}^{t h r}}\right)^{\frac{1}{\alpha_{2}}}+\frac{h}{2},
$$

the proposed schemes are always equal to the conventional schemes. In our network model, such a distance $d$ is about $3660 \mathrm{~m}$.

\section{CONClusions}

In this paper, we considered the distributed power control problem in the CR network which shares the PU channel. We proposed fully autonomous distributed power control algorithms for CR networks that guarantee the QoS requirements for both the PU and SU simultaneously. By adding one more transmission power constraint to the conventional distributed power control algorithms, the proposed schemes ensure the priority of the PU. Specifically, in the previous works, the transmission power of each SU is constrained by the sum of the interference induced by all SUs in the network. Since the amount of total interference cannot be identified by local information of the SU, an additional process is needed to let the SUs recognize the interference temperature at the primary receiver. This additional process is an obstacle to the fully autonomous distributed power control. By translating the constraint for the whole network into the individual one, however, the proposed schemes easily realize the fully autonomous distributed power control. The transmission power constraint of each SU to protect the PU is numerically derived. Through the simulation results, we demonstrated that the proposed schemes absolutely protect the PU.

In the future works, the issues related to the convergence of the proposed scheme will be addressed owing to the importance of the convergence rate of the proposed scheme using iterative method.

\section{REFERENCES}

[1] FCC, "Spectrum Policy Task Force Report," ET Docket No. 02-135, Nov. 2002.

[2] FCC, "Notice of Proposed Rule Making," ET Docket No. 04-113, May 2004.

[3] M. J. Marcus, "Unlicensed Cognitive Sharing of TV Spectrum: The Controversy at the Federal Communications Commission," IEEE Commun. Mag., vol. 43, no. 5, pp. 24-25, May 2005.

[4] S. Haykin, "Cognitive Radio: Brain-Empowered Wireless Communications," IEEE J. Sel. Areas Commun., vol. 23, no. 2, pp. 201-220, Feb. 2005.

[5] J. T. Le and Q. Liang, "An Efficient Power Control Scheme for Cognitive Radios," in IEEE Wireless Communications and Networking Conference, 2007, pp. 2559-2563.

[6] W. Wang, T. Peng, and W. Wang, "Optimal Power Control under Interference Temperature Constraint in Cognitive Radio Network," in IEEE Wireless Communications and Networking Conference, 2007, pp. 116-120.

[7] Y. Xing and R. Chandramouli, "QoS Constrained Secondary Spectrum Sharing," in IEEE Dynamic Spectrum Access Networks, 2005, pp. 658661.

[8] L. Qian, X. Li, J. Attia, and Z. Gajic, "Power Control for Cognitive Radio Ad Hoc Networks," in IEEE Local and Metropolitan Area Networks, 2007, pp. 7-12.

[9] G. J. Foschini and Z. Miljanic, "A Simple Distributed Autonomous Power Control Algorithm and its Convergence," IEEE Trans. Veh. Technol., vol. 42, no. 4, pp. 641-646, Nov. 1993.

[10] S. A. Grandhi, J. Zander, and R. Yates, "Constrained Power Control," Wireless Pers. Commun., vol. 1, no. 4, pp. 257-270, Dec. 1994.

[11] F. Berggren, R. Jantti, and S. Kim, "A Generalized Algorithm for Constrained Power Control With Capability of Temporary Removal," IEEE Trans. Veh. Commun., vol. 50, no. 6, pp. 1604-1612, Nov. 2001.

[12] C. E. Perkins and E. M. Royer, "Ad-hoc On-Demand Distance Vector Routing," in IEEE Mobile Computing Systems and Applications, 1999, pp. 90-100.

[13] S. Mangold, A. Jarosch, and C. Monney, "Operator Assisted Cognitive Radio and Dynamic Spectrum Assignment with Dual Beacons - Detailed Evaluation," in IEEE Communication System Software and Middleware, 2006, pp. 1-6. 